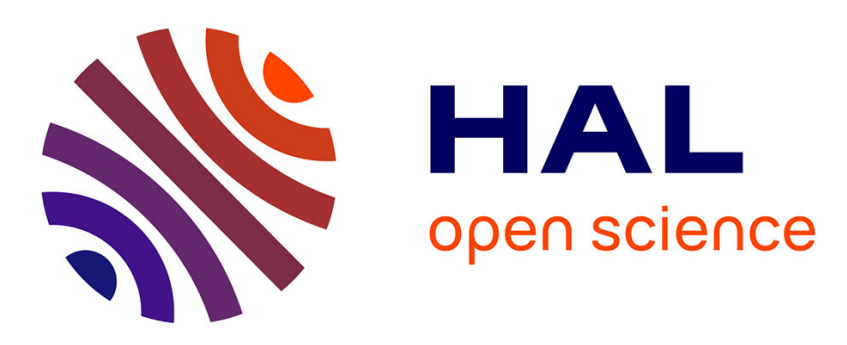

\title{
Optimal Prefiltered Stochastic Transmitted Waves for Fat Inclusion Detection in Milk with Harmonic Ultrasound
}

\author{
Sébastien Ménigot, Nesrine Houhat, Jean Marc Girault
}

\section{To cite this version:}

Sébastien Ménigot, Nesrine Houhat, Jean Marc Girault. Optimal Prefiltered Stochastic Transmitted Waves for Fat Inclusion Detection in Milk with Harmonic Ultrasound. 2019 IEEE International Ultrasonic Symposium, Oct 2019, Glasgow, United Kingdom. 10.1109/ULTSYM.2019.8925979 hal02311265

\section{HAL Id: hal-02311265 \\ https://hal.science/hal-02311265}

Submitted on 10 Oct 2019

HAL is a multi-disciplinary open access archive for the deposit and dissemination of scientific research documents, whether they are published or not. The documents may come from teaching and research institutions in France or abroad, or from public or private research centers.
L'archive ouverte pluridisciplinaire HAL, est destinée au dépôt et à la diffusion de documents scientifiques de niveau recherche, publiés ou non, émanant des établissements d'enseignement et de recherche français ou étrangers, des laboratoires publics ou privés. 


\section{Optimal Prefiltered Stochastic Transmitted Waves for Fat Inclusion Detection in Milk with Harmonic Ultrasound}

\author{
Sébastien Ménigot \\ ESEO Group, \\ Angers, France \\ LAUM, UMR-CNRS 6613, \\ Université du Maine, \\ Le Mans, France \\ sebastien.menigot@eseo.fr
}

\author{
Nesrine Houhat \\ Research Center \\ in Industrial Technologies CRTI, \\ P. O. Box 64, Cheraga, Algiers \\ n.houhat@crti.dz
}

\author{
Jean-Marc Girault \\ ESEO Group, \\ Angers, France \\ LAUM, UMR-CNRS 6613, \\ Université du Maine, \\ Le Mans, France \\ jean-marc.girault@eseo.fr
}

\begin{abstract}
In non destructive testing, improvements have been made possible by taking into account the harmonic frequencies, as in agri-food domain. The transmitted signal are often selected empirically as a fixed-frequency Gaussian pulse, by taking into account the transducer bandwidth only. However, waveform should take into account all the features of the ultrasound system and of the medium. To design the waveform, a genetic algorithm looks for the best stochastic wave. However, one of limitations in this optimization process is the high amount of transmitted waves. To reduce this number, instead of transmitting wide band stochastic waves, narrow band stochastic waves limited by the transducer bandwidth are preferred. The optimization was thus applied on the detection of fat cluster in milk by maximizing the signal-to-noise ratio (SNR), while decreasing the amount of transmitted waves. Twelve combinations from different limited bandwidths of transmitted waves were tested. Whereas the low cut-off frequencies did not change the performances, the high cut-off frequencies affected the convergence speed. In this study, it is shown that the best optimization was twelve times faster with the high cut-off frequency of $5.6 \mathrm{MHz}$ and led to a gain of $62 \%$ compared to the SNR obtained with a best fixed-frequency sine wave.

Index Terms-Nonlinearity, optimal wave, genetic algorithm
\end{abstract}

\section{INTRODUCTION}

Nonlinear ultrasound systems have improved the structure/inclusion detection in fluids by enhancing the level of backscattered harmonics. This is particularly the case for detecting fat clusters in the agri-food domain [1], i.e. in cheese manufacturing. Its simple principle consists in transmitting an ultrasound sinus wave of frequency $f_{0}$ into the medium being explored and in receiving harmonic components $\left(2 f_{0}, 3 f_{0}, \ldots\right)$ generated during the wave propagation. Several methods of harmonic extraction have been developed by filtering the echoes or by coding successive transmitted waves [2]. The second kind of methods have improved the signal-to-noise ratio (SNR) while ensuring a good axial resolution, such as pulse inversion imaging [3]. Since the most commonly used is the pulse inversion imaging, we only focused our study with this technique.
Even if most of improvements includes post-processing to extract harmonics, the choice of the transmitted wave is decisive. However, the usual settings of the transmitted wave are empirically constrained to a fixed-frequency sine wave, because the optimal settings requires knowledges experimentally inaccessible about the transducer and the fluid. A first solution carries in a suboptimal solution by finding the parameter of the waveform, such as frequency [4]. However, this optimization problem can be solved in an optimal way by sending stochastic waves iteratively, without any assumption on the waveform [5].

In this study in the nonlinear imaging context using pulse inversion, a genetic algorithm looks for the best stochastic wave by optimizing the SNR between a reference (milk without any fat cluster) and fat cluster as elements in the milk. One of limitations in this optimization process is the high amount of transmitted waves required to reach the optimal SNR enhancing the fat cluster. To reduce this amount, instead of transmitting wide band stochastic waves by the transducer, stochastic waves were prefiltered by a narrow band filter similar to the transducer bandwidth. Here, we propose to break the principle of not taking into account any a priori information by proposing a prefiltering based on the spectral parameters of the transducer in an acceleration of the optimization process, while reaching the global optimum.

\section{Optimization with Prefiltered Stochastic WAVES}

Finding the best prefiltered stochastic waveform $\mathrm{w}_{\text {filtered }}$ is a hard optimization problem, which is more complex with the nonlinear imaging context using pulse inversion. To solve it, a pulse inversion system is modified as an iterative close loop system including a metaheuristics in the feedback. Among the metaheuristics, genetic algorithm [6] can optimize a cost function by selecting parameters concatenated in a vector. Therefore, it seems to be adjusted to our problem, since 
prefiltered stochastic waveforms can be written as a vector containing $N_{s}$ samples. The problem can be simply written:

$$
\mathbf{w}_{\text {filtered }}^{\star}=\arg \max _{\mathbf{w}_{\text {filtered }}}(\mathrm{SNR}),
$$

where cost-function allows us to enhance the backscattered power of fat cluster $P_{\text {fat }}$ cluster in comparison with the backscattered power of the surrounding milk $P_{\text {milk }}$, such as:

$$
S N R=10 \log _{10}\left(\frac{P_{\text {fat } \text { cluster }}}{P_{\text {milk }}}\right) .
$$

First, following the procedure of the genetic algorithm [6], 12 prefiltered stochastic waveforms $\mathbf{w}_{\text {filtered }}$ are randomly generated from white noise (magnitude between -1 and 1) by the genetic algorithm. They are prefiltered by a finite impulse response (FIR) filter where their bandwidths at $-3 \mathrm{~dB}$ are between the low cut-off frequency $f_{\text {low }}$ and the high cut-off frequency $f_{\text {high }}$ [7]. Before the transmission, the power of the prefiltered stochastic waveforms $\mathbf{w}_{\text {filtered }}$ are normalized by adjusting the amplitude $A$, so that They are constant to the power $P_{x_{r e f}}$ of the impulse response of the transducer driving pressure $A_{0}$ :

$$
\mathbf{x}_{q}=(-1)^{q} \cdot A \cdot \mathbf{w}_{\text {filtered }},
$$

with $q=1,2$ and

$$
A=\sqrt{\frac{A_{0}^{2} \cdot P_{x_{\text {ref }}}}{\mathbf{w}_{\text {filtered }}^{T} \cdot \mathbf{w}_{\text {filtered }}}},
$$

where $w^{T}$ denotes the transpose of the matrix $w$. Note that the number of samples $N_{s}$ of each waveform is set so that the duration $T$ of the stochastic waveform $\mathbf{w}$ corresponded to $100 \%$ of the fractional bandwidth of the transducer. Finally, the transmitted and prefiltered stochastic waves $\mathbf{x}_{1}$ and the wave with their opposite phases $\mathbf{x}_{2}\left(\mathbf{x}_{1}=-\mathbf{x}_{2}\right)$ are sequentially transmitted into the milk including fat cluster.

Second, for each echoes of the 12 waveform couples, even harmonic components are respectively extracted by the sum $\mathbf{z}$ of their two respective echoes $\mathbf{y}_{1}$ and $\mathbf{y}_{2}$. The 12 respective SNR are assessed on that RF line $\mathbf{z}$.

Finally, a genetic algorithm generates a new prefiltered stochastic waveform $\mathbf{w}$ to optimize the SNR. Among the 12 prefiltered stochastic waveforms, only the 6 best individual waveforms which maximized the SNR are selected. To construct them, the crossover operator mixed the best previous waveform with one of the 5 remaining better waveforms. Finally, $40 \%$ samples were mutated to obtain robust optimization. Moreover, the 12 new stochastic waveform are prefiltered by the FIR filter to guarantee their bandwidth.

\section{Simulation Model}

As described previously, the prefiltered stochastic waves $\mathbf{x}$ and their opposite phases were generated digitally with Matlab (Mathworks, Natick, MA, USA). They were transmitted by a transducer centred at $4 \mathrm{MHz}$ with a bandwidth of $80 \%$ at $-3 \mathrm{~dB}$

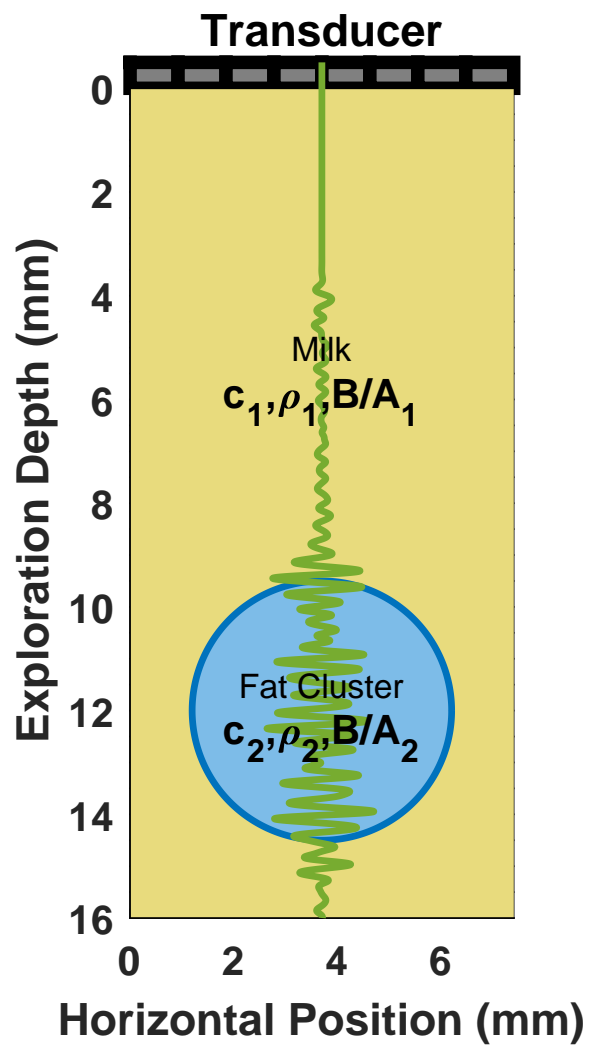

Fig. 1. Grid of properties of the medium constituted of milk (medium 1) with a fat cluster (medium 2): $c$ is the speed of sound wave velocity, $\rho$ the density and $B / A$ the nonlinearity parameter. The ultrasound transducer was at a depth of $0 \mathrm{~mm}$, here at the top. As an illustration, a harmonic RFline $\mathbf{z}$ is depicted in green.

$(2.4-5.6 \mathrm{MHz})$ and $200 \%$ at $-10 \mathrm{~dB}(0.25-14 \mathrm{MHz})$. The pulse waves generated were sequentially propagated nonlinearly into the attenuating medium of milk [8]. In the milk, a 4 $\mathrm{mm}$-diameter fast cluster was inserted into the milk at $12 \mathrm{~mm}$ below the surface (Fig. 1). Note that the medium properties are shown in table I. Finally, the echoes were filtered by the same transducer than this one in transmission. The genetic algorithm proposes new prefiltered stochastic waves and the procedure is repeated until the end of the optimization.

\section{RESULTS}

The optimization process was tested with 12 combinations from different limited bandwidths of transmitted waves (4 low and 3 high cut-off frequencies). The low and the high cutoff frequencies are listed in table II. Note that these values were chosen from the cut-off frequencies of the transducer. Each setting of bandwidth is repeated 10 times, in order to remove the random effect of the algorithm and to check if the optimization converges to the same value. Note that the other settings were always the same:

- the driving pressure $A_{0}$ was set to $400 \mathrm{kPa}$;

- the waveform was described by $N_{s}=40$ samples, since its duration had to represent $100 \%$ of the fractional bandwidth of the transducer. 
TABLE I

Mechanical Properties of EACH EXPLORED. N IS THE Symbol of the GaUSSIAN Distribution.

\begin{tabular}{lll}
\hline & Milk [9] & Fat Cluster [10] \\
\hline Speed of sound $c$ & $\mathcal{N}\left(1531 \mathrm{~m} / \mathrm{s}, 0.008 \mathrm{~m}^{2} / \mathrm{s}^{2}\right)$ & $\mathcal{N}\left(1060 \mathrm{~m} / \mathrm{s}, 75 \mathrm{~m}^{2} / \mathrm{s}^{2}\right)$ \\
Density & $\mathcal{N}\left(1025 \mathrm{~kg} / \mathrm{m}^{3}, 0.008 \mathrm{~kg}^{2} / \mathrm{m}^{6}\right)$ & $\mathcal{N}\left(928 \mathrm{~kg} / \mathrm{m}^{3}, 75 \mathrm{~kg}^{2} / \mathrm{m}^{6}\right)$ \\
Nonlinearity parameter $B / A$ & 5.1 & 10.3 \\
\hline
\end{tabular}

TABLE II

LIST OF LOW AND 4 HIGH CUT-OFF FREQUENCIES OF THE PREFILTERED STOCHASTIC WAVEFORM. NOTE THAT THE CUT-OFF FREQUENCIES OF THE TRANSDUCER ARE WRITTEN IN BOLD

\begin{tabular}{lllll}
\hline Low cut-off freq. & $0.44 \mathrm{MHz}$ & $0.88 \mathrm{MHz}$ & $1.17 \mathrm{MHz}$ & $\mathbf{2 . 4} \mathbf{~ M H z}$ \\
\hline High cut-off freq. & $\mathbf{5 . 6} \mathbf{M H z}$ & $11 \mathrm{MHz}$ & $17 \mathrm{MHz}$ & \\
\hline
\end{tabular}

Moreover, as an illustration, the results were compared to a standard fixed-frequency Gaussian pulse for which its frequency was set to optimize the SNR.

First, the low cut-off frequency of the prefiltered waveform was fixed at the same value than the low cut-off frequency of the transducer, i.e. $2.4 \mathrm{MHz}$. The high cut-off frequency of the prefiltered waveform was changed to the value described in table II. Note that these values was chosen to have at least the same bandwidth of transducer at $-3 \mathrm{~dB}$ and thus to keep the spatial resolution. Fig. 2 show the optimization of the SNR as a function of the genetic algorithm iteration for the 3 high cut-off frequencies of the prefiltered waveform. Whatever the high cut-off frequency, the SNR increased during the genetic algorithm iterations. At the end of the optimization process, the SNR was increased by $3 \mathrm{~dB}$ in comparison with the SNR obtained with a best fixed-frequency Gaussian pulse. Moreover, the maximal SNR were higher by using prefiltering waveform than the optimization without prefiltering. However, the narrower bandwidth allowed us to reach better SNR. Finally, with the better prefilter settings (a high cut-off frequency of $5.6 \mathrm{MHz}$ ), the convergence was quicker, since a SNR of $8.25 \mathrm{~dB}$ can be reach with only 4 iterations, compared to the 12 iterations required without prefiltering.

Second, the high cut-off frequency of the prefiltered waveform was fixed at the same value than the high cut-off frequency of the transducer, i.e. 5.6 MHz. The low cut-off frequency of the prefiltered waveform was changed to the value described in table II. Fig. 3 show the optimization of the SNR as a function of the genetic algorithm iteration for the 4 low cut-off frequencies of the prefiltered waveform. Whatever the low cut-off frequency, the SNR increased during the genetic algorithm iterations. At the end of the optimization process, the SNR was increased by $3.4 \mathrm{~dB}$ in comparison with the SNR obtained with the best standard fixed-frequency Gaussian pulse. Moreover, the maximal SNR were always higher by using prefiltering waveform than the optimization without prefiltering and than the waveform prefiltered with the high cut-off frequencies described in Fig. 2. However, whatever the low cut-off frequency, the SNR is equivalent. Nevertheless, a lower cut-off frequency slightly smaller than the cut-off frequency of the transducer makes it possible

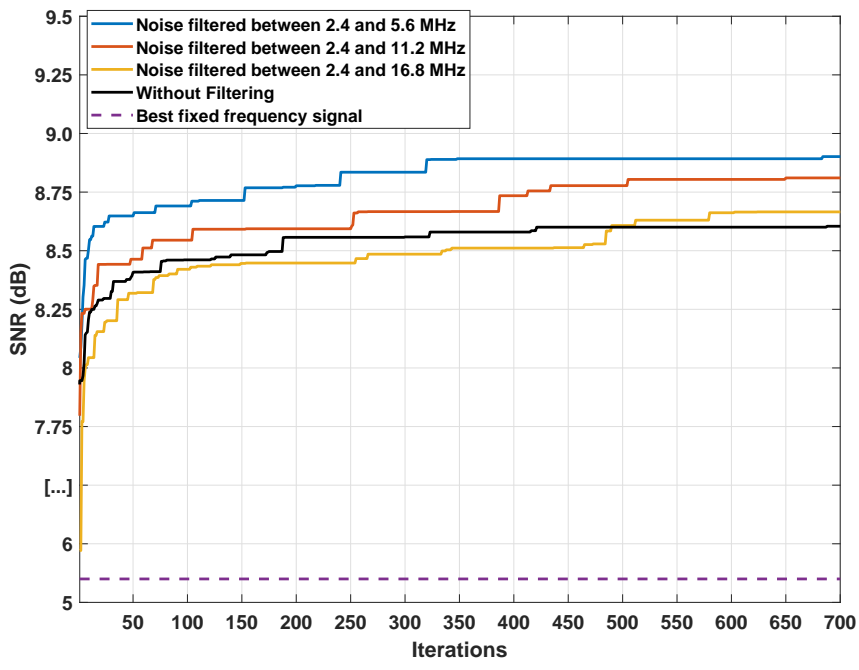

Fig. 2. Median of optimized SNR from 10 simulations as a function of the genetic algorithm iteration for different high cut-off frequencies of the prefiltered waveform. Note that the SNR value shown here are the median for 10 simulations in the same conditions. The low cut-off frequency of the prefiltered waveform is fixed at the same value than the low cut-off frequency of the transducer, i.e. $2.4 \mathrm{MHz}$. As an illustration, the optimization without any constraint on the bandwidth is depicted in black. Moreover, the performance using the best fixed-frequency Gaussian pulse is depicted in dash line.

TABLE III

NUMBER ITERATIONS REQUIRED TO REACH 8.5 DB WITH THE GENETIC ALGORITHM AND THE MAXIMAL SNR AT THE END OF THE OPTIMIZATION PROCESS.

\begin{tabular}{cccc}
\hline $\begin{array}{c}\text { Low cut-off } \\
\text { frequency }\end{array}$ & $\begin{array}{c}\text { High cut-off } \\
\text { frequency }\end{array}$ & $\begin{array}{c}\text { Iterations } \\
\text { to reach } \mathbf{8 . 5} \mathbf{~ d B}\end{array}$ & $\begin{array}{c}\text { Maximal } \\
\text { SNR }\end{array}$ \\
\hline $0.88 \mathrm{MHz}$ & $5.6 \mathrm{MHz}$ & 15 & $8.9 \mathrm{~dB}$ \\
None & None & 187 & $8.6 \mathrm{~dB}$ \\
\hline
\end{tabular}

to statistically obtain a slightly higher SNR. Note that this improvement was not significant for the 40 simulations (4 settings repeated 10 times).

To summarize, table III shows the iterations to reach a SNR of $8.5 \mathrm{~dB}$ and the maximal SNR at the end of the optimization process. As shown in Figs. 2 and 2, the optimization process with prefiltering can be about 12 times faster (187/15). After only one iteration, the SNR obtained by prefiltering stochastic waveform was higher than this one obtained by stochastic waveform without prefiltering and by the best fixed-frequency Gaussian pulse. Moreover, after only 6 iterations, the SNR was increased by $3 \mathrm{~dB}$ compared to the SNR obtained with the best fixed-frequency Gaussian pulse. Finally, at the end of the optimization process, the maximal SNR was higher with prefiltered stochastic waveform than without prefiltering. 


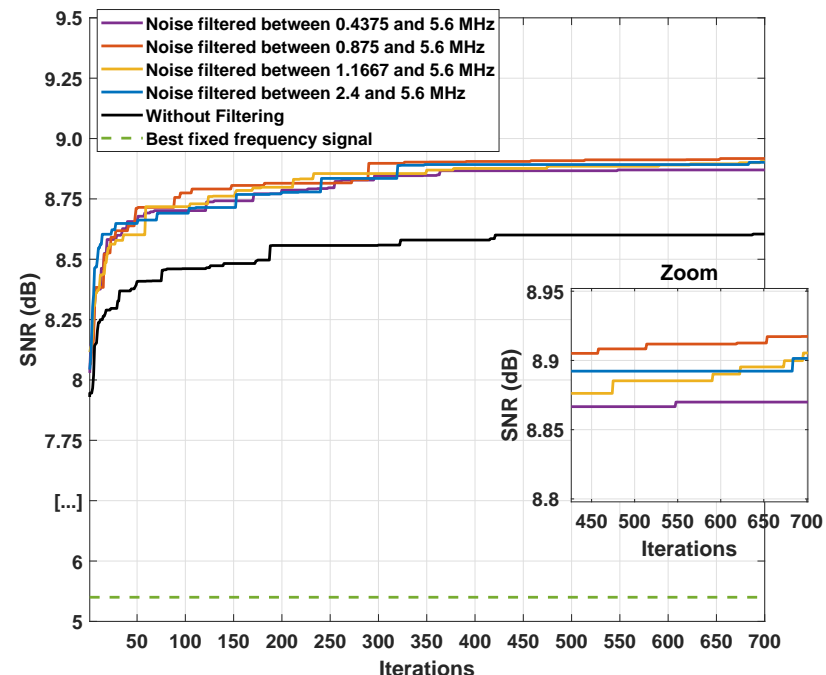

Fig. 3. Median of optimized SNR from 10 simulations as a function of the genetic algorithm iteration for different low cut-off frequencies of the prefiltered waveform. Note that the SNR value shown here are the median for 10 simulations in the same conditions. The high cut-off frequency of the prefiltered waveform is fixed at the same value than the high cut-off frequency of the transducer, i.e. $5.6 \mathrm{MHz}$. As an illustration, the optimization without any constraint on the bandwidth is depicted in black. Moreover, the performance using the best fixed-frequency Gaussian pulse is depicted in dash line.

However, note that the genetic algorithm should be reach the maximal SNR without prefiltering, but it would require a higher number of iterations.

As an illustration, Fig.4a shows the optimal transmitted stochastic wave $\mathbf{x}$ obtained by the genetic algorithm at the transducer output when the prefiltered stochastic waveform $\mathbf{w}$ is optimal. Note that this signal was propagated in the milk. The harmonic echoes $\mathbf{z}$ obtained by using the optimal transmitted stochastic wave $\mathbf{x}$ is shown in Fig.4b. As expected, it was higher to the harmonic echoes obtained by using the better fixed-frequency Gaussian pulse (shown in red).

\section{Discussion AND CONCLUSIONS}

Prefiltered stochastic waveform were automatically selected in order to optimize the SNR assessed in a pulse inversion imaging system to detect fat cluster in milk. To help the optimization process, the stochastic waveform were prefiltered to constrain the research space. Thus the SNR could be increased by $5 \%$ compared to the optimization without prefiltering and $62 \%$ compared to a fixed-frequency Gaussian pulse.

However, the settings of the cut-off frequencies had an influence on the better SNR obtained after optimization. First, the high cut-off frequency had to be close to the cut-off frequency of the transducer. If the high cut-off frequency were higher than the high cut-off frequency of the transducer, the harmonic generated during the propagation could be detected, because out of bandwidth. Second, the low cut-off frequency had no effect on the better SNR. Therefore, the best prefilter for stochastic waveform is a low-pass prefilter where its cut-off frequency is the high cut-off frequency.
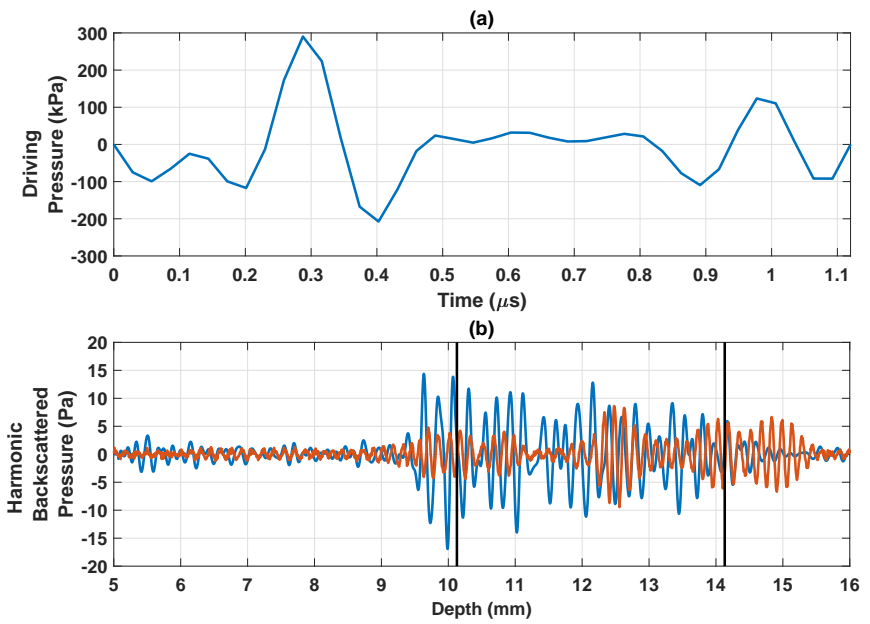

Fig. 4. (a) Optimal transmitted stochastic wave $\mathbf{x}$ obtained by the genetic algorithm at the transducer output when the prefiltered stochastic waveform w is optimal. (b) Harmonic backscattered pressure obtained by pulse inversion and optimal prefiltered waveform. As an illustration, the harmonic RF line in red is obtained by using the better fixed-frequency Gaussian pulse.

Finally, as the optimization is done in a small area by taking the transducer features into account, the genetic algorithm looks twelve times faster for the optimal waveform. Moreover, the optimization including a prefiltering makes possible to obtain higher SNR than an optimization without prefiltering, in just one iteration.

In conclusion, this method improved the SNR without strong assumptions on the waveform. This settings should be suitable to find more easily the optimal waveform in non destructive testing application.

\section{REFERENCES}

[1] H. Feng, G. V. Barbosa-Canovas, and J. Weiss, Ultrasound technologies for food and bioprocessing. New York, NY, USA: Springer, 2011.

[2] P. N. Burns, D. H. Simpson, and M. A. Averkiou, "Nonlinear imaging," Ultrasound in Medicine and Biology, vol. 26, pp. S19-S22, 2000.

[3] D. H. Simpson, C. T. Chin, and P. N. Burns, "Pulse inversion doppler: A new method for detecting nonlinear echoes from microbubble contrast agents," IEEE T. Ultrason. Ferr, vol. 46, no. 2, pp. 372-382, Mar. 1999.

[4] S. Ménigot, J.-M. Girault, I. Voicu, and A. Novell, "Optimization of contrast to tissue ratio by frequency adaptation in pulse inversion imaging," IEEE Trans Ultrason Ferroelectr Freq Control, vol. 59, no. 11, pp. 2431-2438, Nov. 2012.

[5] S. Ménigot and J.-M. Girault, "Optimization of contrast resolution by genetic algorithm in ultrasound tissue harmonic imaging," Ultrasonics, vol. 71, pp. 231-244, 2016.

[6] R. L. Haupt and S. E. Haupt, Practical Genetic Algorithms, 2nd ed. Hoboken, NJ, USA: John Wiley \& Sons Inc, Jun. 2004.

[7] J. O. Smith, Spectral audio signal processing. Stanford, CA, USA: W3K Publishing, 2011.

[8] M. E. Anderson, "A 2d nonlinear wave propagation solver written in open-source matlab code," in Proceeding IEEE Ultrasonic Symposium, San Juan, Puerto Rico, Oct. 2000, pp. 1351-1354.

[9] X.-F. Gong, Z.-M. Zhu, T. Shi, and J.-H. Huang, "Determination of the acoustic nonlinearity parameter in biological media using fais and itd methods," The Journal of the Acoustical Society of America, vol. 86, no. 1, pp. 1-5, 1989.

[10] T. Szabo, Diagnostic Ultrasound Imaging: Inside Out. London, UK: Academic Press, 2004. 